Abstract

\title{
Weak Isospin Symmetry and the Vacuum Polarization Energy of Cosmic Strings ${ }^{\dagger}$
}

\author{
Herbert Weigel \\ Institute for Theoretical Physics, Physics Department, Stellenbosch University, Matieland 7602, South Africa; \\ weigel@sun.ac.za \\ † Presented at Symmetry 2017-The First International Conference on Symmetry, Barcelona, Spain, \\ 16-18 October 2017. \\ Published: 3 January 2018
}

The vacuum polarization energy is the leading quantum correction to the energy of a localized field configuration. Spectral methods are an effective means to calculate vacuum polarization energies [1]. When computing these energies for cosmic strings in models similar to the standard model of particle physics, regularization and renormalization requires operations that individually are not manifestly consistent with the isospin symmetry of the model. Formally, i.e., before regularization and renormalization, the vacuum polarization energy is defined via the single particle energies of the quantum fluctuations that interact with a background potential which is generated by the cosmic string. Fortunately, there exists a particular global isospin transformation of the string configuration that leaves the single particle energies invariant. By numerical simulations, it is thus possible to verify that the renormalized vacuum polarization energy computed by spectral methods indeed preserves isospin symmetry. The particular construction even confirms that the spectral methods approach is consistent with local isospin symmetry [2]. These findings strongly support the use of spectral methods to compute vacuum polarization energies. It is then shown that the quantum corrections lead to stabilization of a cosmic string when the parameters of the model are slightly changed from their standard model values.

\section{References}

1. Graham, N.; Quandt, M.; Weigel, H. Spectral Methods in Quantum Field Theory; Lecture Notes in Physics; Springer: Berlin, Heidelberg, 2009; Volume 777, p. 1.

2. Weigel, H.; Quandt, M.; Graham, N. Isospin invariance and the vacuum polarization energy of cosmic strings. Phys. Rev. D 2016, 94, 045015.

(C) 2018 by the author. Licensee MDPI, Basel, Switzerland. This article is an open access article distributed under the terms and conditions of the Creative Commons Attribution (CC BY) license (http://creativecommons.org/licenses/by/4.0/). 